\title{
Investigating a solar influence on cloud cover using the North American Regional Reanalysis data
}

\author{
Daniel Scott Krahenbuhl* \\ School of Geographic Sciences and Urban Planning, Arizona State University, Tempe, AZ 85287-5302, USA \\ *Corresponding authors: daniel.krahenbuhl@asu.edu
}

Received 6 March 2015 / Accepted 20 April 2015

\begin{abstract}
The controversial connection between cosmic rays, solar activity, and cloud cover is investigated using a climatological reconstructed reanalysis product: the North American Regional Reanalysis which provides high-resolution, low, mid-level, high, and total cloud cover data over a Lambert conformal conic projection permitting land/ocean discrimination. Pearson's product-moment regional correlations were obtained between monthly cloud cover data and solar variability indicators, cosmic ray neutron monitors, several climatological indices, including the Atlantic Multidecadal Oscillation (AMO), and between cloud layers. Regions of the mid-latitude oceans exhibited a positive correlation with cosmic ray flux. Additionally, this maritime low cloud cover exhibits the only failed correlation significance with other altitudes. The cross correlation reveals that cloud cover is positively correlated everywhere but for ocean low cloud cover, supporting the unique response of the marine layer. The results of this investigation suggest that with the assumption that solar forcing does impact cloud cover, measurements of solar activity exhibits a slightly higher correlation than GCRs. The only instance where GCRs exhibit a positive regional correlation with cloud cover is for maritime low clouds. The AMO exerts the greatest control of cloud cover in the NARR domain.
\end{abstract}

Key words. Solar activity - Clouds - Atmosphere - Cosmic ray - Climate

\section{Introduction}

Solar variability has long been suspected as having a significant influence on climate change. Solar cycles of magnetic variability result in changes in the luminal spectra of the sun, the properties of the solar wind, and the flux of galactic cosmic rays incident on Earth's atmosphere. Changes in the total solar irradiance (TSI) with enhanced extreme ultraviolet (EUV) emission have been hypothesized to induce a dynamic air/ ocean circulation response resulting from stratospheric ozone production (Lean \& Rind 1998). The configuration of the interplanetary magnetic field (IMF) deflects and entrains galactic cosmic radiation (GCR) that transits the sphere of influence controlled by the sun, i.e., the heliosphere. During periods of heightened solar activity, fewer galactic cosmic rays (GCRs) are detected by earthbound neutron monitors. GCR flux and its correlation with cloud cover remain the most controversial of potential variables influencing the climate system.

This study will examine the strength of relationship between GCR flux, solar activity, and a detailed regional cloud cover reanalysis, the North American Reanalysis (Mesinger et al. 2006), a historical climate reanalysis that extends from January 1979 to present. The high resolution of this reanalysis, an accompanying land/ocean mask, and multiple altitudes of cloud cover differentiation, provide an interesting perspective to previous research which utilized direct satellite data.

Svensmark \& Friis-Christensen (1997) purported a controversial correlation between GCR flux and cloud cover. Using cloud cover data from the International Satellite Cloud Climatology Project (ISCCP) and neutron monitors, they report that total cloud cover over the mid-latitude oceans exhibits a positive correlation with GCRs. Hypothetically, an energetic particle shower caused by a GCR impact would cause ionization, resulting in an increase in cloud condensation nuclei $(\mathrm{CCN})$. Periods of anomalously low solar activity, resulting in greater cosmic ray flux and increased cloud cover, would reduce the global temperature (Svensmark 2000).

The Cosmics Leaving Outdoor Droplets (CLOUD) experiment at the European Organization for Nuclear Research (CERN) was able to duplicate the production of $\mathrm{CCN}$ under controlled laboratory conditions (Kirkby et al. 2011), whereby GCRs increase the production rate of condensation nuclei which leads to an abundance of $\mathrm{CCN}$, and the development of cloud droplets (Yu 2002). Their results indicate the mechanism is plausible; however, they also raise doubts as to the magnitude of the effect. Additionally, CLOUD results indicate that the processes are most pronounced in the mid-troposphere and above. Erlykin et al. (2013) review the relevance of the CLOUD experiment, and conclude that GCRs do not affect atmospheric clouds significantly. Additionally, climatological models, which incorporate CCN generation mechanisms and cloud microphysics, fail to produce significant change in global-scale CCN populations, cloud optical properties, or radiative forcing (Snow-Kropla et al. 2011; Dunne et al. 2012; Kazil et al. 2012).

The findings of Svensmark \& Friis-Christensen (1997) and Marsh \& Svensmark (2000) have been met with considerable criticism (Laut 2003). Udelhofen \& Cess (2001) find indications that cloud cover anomalies over the US exhibit a strong coherence with solar activity and not GCR flux. Instead they 
argue that the cloud cover anomalies are an effect of the dynamic circulation response through EUV ozone production. These conclusions are mirrored by Kristjansson et al. (2002) who note falling correlations between low cloud cover and cosmic rays after 1993, leading them to the conclusion that cloud cover correlations are the result from EUV stratospheric ozone production affecting the propagation of planetary waves. Balling \& Cerveny (2003) using the US radiosonde network, find a statistically significant inverse correlation between GCR flux and low cloud cover over the continental United States. Additionally, other studies find no statistically significant correlations between GCR flux and cloud cover (Erlykin et al. 2009a, 2009b, 2009c; Laken et al. 2012a).

Discrimination between radiation effects of clouds at different altitudes is not trivial. For instance, with regard to climate radiative forcing, an increase in thin high cloud cover results in a net warming, whereas increased thick low altitude cloud cover results in a net cooling. Ionized particle production itself is dependent upon altitude. GCR flux causes greater particle production in the low altitudes, and less at higher altitudes. This is particularly notable over the marine layer (Yu 2002).

Unfortunately, cloud cover height discriminations are not uniformly applied between datasets. Initially, low cloud cover was determined to be positively correlated with GCR flux, while high cloud cover exhibited a negative correlation (Marsh \& Svensmark 2000). Additionally, there exist cross correlations between cloud layers themselves (Erlykin et al. 2010). There have been marked criticisms citing satellite sensor limitations of detection between cloud layers and satellite field of view edge effects (Palle 2005; Laken et al. 2012a).

Cloud cover is a climatological field variable having both dimensions of time and space. Consequently, as part of the atmospheric continuum, it exhibits high temporal and spatial autocorrelation. Most of low cloud variability comes from the subtropical oceans, where the bulk of the Earth's low clouds are formed (Kristjansson et al. 2004). Voiculescu et al. (2006) found a positive correlation between low cloud cover variability with GCR flux over ocean regions and dry continental air masses, and a negative correlation between high cloud cover variability with GCR flux, specifically over the oceans and moist continental areas. Usoskin \& Kovaltsov (2008) find strong statistically significant correlations between low clouds and GCR flux in limited geographical regions: the Atlantic Ocean and Europe. Voiculescu \& Usoskin (2012) stressed the importance of examining the correlation between GCR flux and cloud cover, at the regional level, specifically at climate defining regions. However, Laken \& Čalogović (2013) note that a decreased sample size increases the signal-to-noise ratio, limiting the probability of detecting a solar signal. Other studies have indicated that synoptic scale changes in cloud cover are more likely correlated with regional climatological modes or oscillations rather than GCRs (Laken et al. 2012a).

GCR flux and cloud cover have been investigated over shorter timescales, most notably during Forbush Decrease (FD) events, or periods when a solar eruption disrupts the solar wind, causing a temporary reduction in GCRs lasting for a few days. However, Forbush decreases are also accompanied by EUV enhanced TSI and energetic solar proton events, resulting in conflicting effects (Laken et al. 2011; Laken \& Kniveton 2011). Using MODIS data, Kristjannson et al. (2008) find only a modest correlation with cloud cover variability during FDs.
Others find no change in global cloud cover during FD (Čalogović et al. 2010; Laken \& Čalogović 2011).

This investigation extends the previous cloud cover correlations with solar activity galactic cosmic ray flux utilizing the North American Regional Reanalysis (NARR); a high-resolution climatological reconstruction of North America and surrounding areas (Mesinger et al. 2006). Importantly, the NARR uses assimilated weather observations, which provide gridded cloud cover percentages at altitudes similar to ISCCP and MODIS datasets. Additionally, the NARR comes with a land/sea mask for quick differentiation between maritime and continental cloud cover. Additionally, several climatological oscillatory mode data will be used as controls to assess the correlations between GCR flux, solar activity, and cloud cover: specifically the Atlantic Multidecadal Oscillation (AMO), the multivariate El Nino Southern Oscillation (ENSO), the North Atlantic Oscillation (NAO), the Pacific Decadal Oscillation (PDO), and the Quasi-Biennial Oscillation (QBO).

\section{Data}

The North American Regional Reanalysis is a climatological combined model and assimilated dataset covering 1979 to present at $3 \mathrm{~h}$ resolution. It is a supplement to the NCEP/ NCAR reanalysis and is structured on a high-resolution grid on a Northern Hemisphere Lambert Conformal Conic projection. The reanalysis includes cloud cover data for low $(p>642 \mathrm{mb})$, mid-level (642-350 mb), high $(p<350 \mathrm{mb})$, and total cloud cover.

The NARR uses the cloud prediction scheme from the ETA model developed by NCAR (Zhao et al. 1997). The model uses atmospheric moisture variables (specific humidity) for its cloud prediction schemes. The cloud predictions are incorporated into the model's radiation calculations. The data are assimilated, passed through the model physics core, providing a fresh re-parameterized grid, insulating it from errors that might arise from satellite observations, sensor failures, or field of view biases. The NARR effectively provides an additional "frame of reference" to complement previous investigations utilizing first-order remotely sensed products. Reanalysis products are essentially "massaged" data and it is possible that if solar signals ever existed in the observational data, they could have been entirely removed by models during the creation of the reanalysis data.

The NARR data was obtained via PSD ESRL Physical Science Division at the Earth Systems Research Laboratory in Boulder, Colorado (http://www.esrl.noaa.gov/psd/data/narr/). Monthly mean data was used along with the long-term monthly means. To distinguish between ocean cloud layers and land cloud cover, NARR land/ocean mask was also obtained.

Monthly sun spot numbers (1979-2013) were obtained from the Marshal Space Flight Center, NASA (http:// solarscience.msfc.nasa.gov/). Solar $10.7 \mathrm{~cm}$ radio flux $\left(F_{\mathrm{s}}\right)$ monthly means (1979-2013) were obtained from via PSD ESRL Physical Science Division at the Earth Systems Research Laboratory in Boulder, Colorado. Climate indices for the Atlantic Multidecadal Oscillation (AMO), the multivariate El Nino Southern Oscillation (ENSO), the North Atlantic Oscillation (NAO), the Pacific Decadal Oscillation (PDO), and the Quasi-Biennial Oscillation (QBO) were also obtained via the PSD ESRL. 


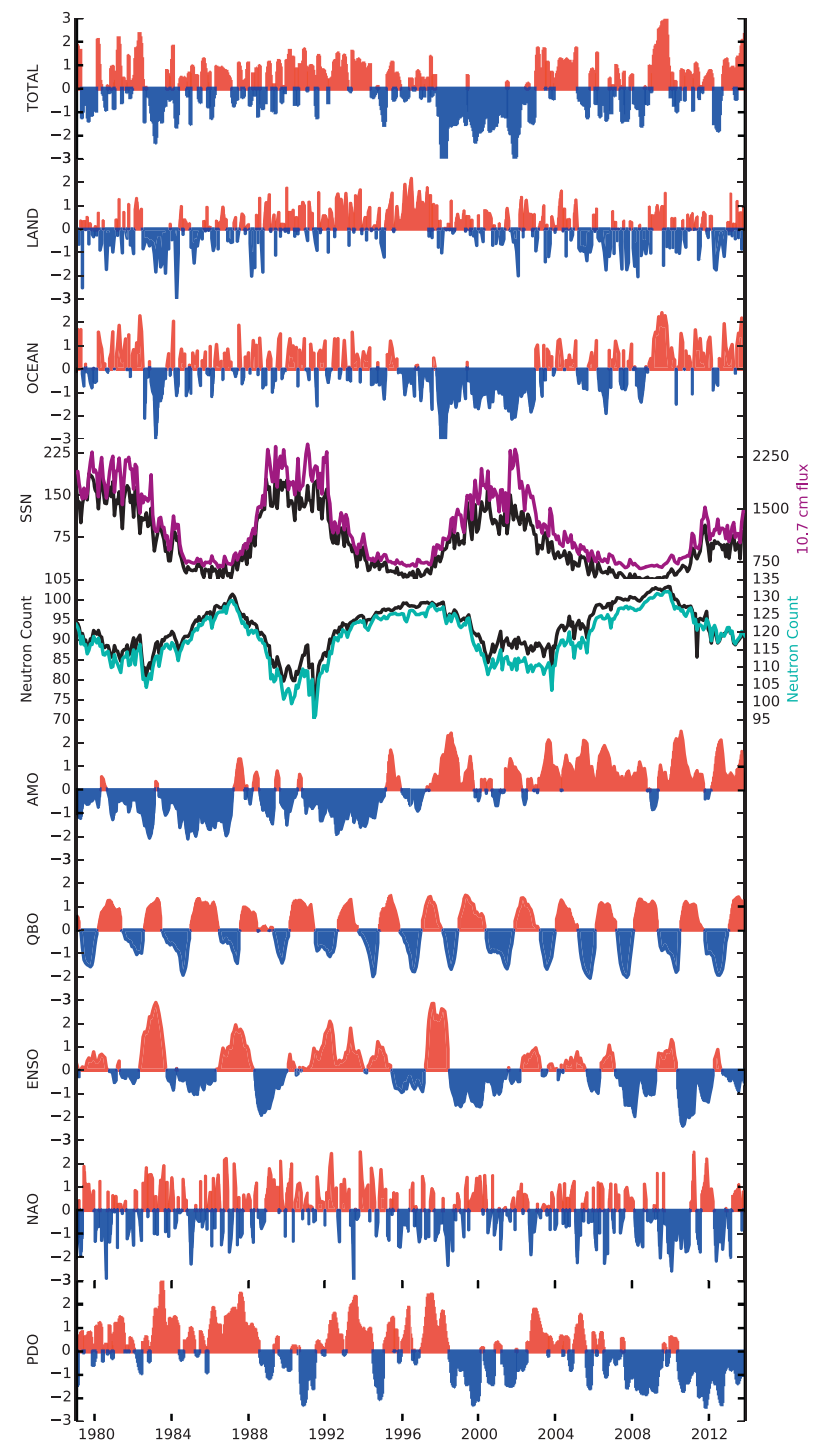

Fig. 1. Time series utilized for this investigation from January 1979 to December 2013 at monthly resolution. Total cloud cover detrended standardized anomalies averaged over the entire NARR domain; total cloud cover detrended standard anomalies averaged over continental landmass; total cloud cover detrended standard anomalies averaged over oceans; sun spot number and $10.7 \mathrm{~cm}$ solar radio flux; GCR neutron monitors; the Atlantic Multidecadal Oscillation; the Quasi-Biennial Oscillation; the Multivariate El Nino Southern Oscillation; the North Atlantic Oscillation; and the Pacific Decadal Oscillation.

North American GCR neutron data was obtained via the Neutron Monitor Database (http://www.nmdb.eu). Stations for Newark, New Jersey (NM1) and Thule Air Force base, Greenland (NM2), were selected for their continuous monthly observations matching the NARR time domain, 1979 through 2013. Total cloud cover data for land, ocean, and total, as well as time series for solar proxies (SSN, FX), GCR data (NM1, NM2), and climate oscillation time series, are depicted in Figure 1.

\section{Methodology}

Deseasonalized, detrended cloud cover standardized anomalies were obtained by applying a 12-month boxcar moving average for the respective cloud levels (low, medium, high, and total). A two-tailed Pearson's product-moment $(r)$ correlation test was applied to every gridpoint in the NARR field for low level, mid-level, high level, and total cloud cover, versus the solar activity proxies (SSN and Fs), the GCR proxies (NM1, $\mathrm{NM} 2$ ), and the climatological indices (AMO, QBO, ENSO, $\mathrm{NAO}$, and PDO). Regional correlations were obtained by averaging correlation coefficients which exhibit $p$-values less than 0.05 , for land, ocean, and total field domains. The correlation tests are only performed with a zero lag, under the assumption that atmospheric ionization and cloud cover responses operate at sub-monthly timescales (Arnold 2006). The time series with $n=420$ values provides 418 degrees of freedom, yielding a critical correlation coefficient of $0.096\left(r_{\text {critical }}\right)$ at a $p$-value of 0.05 . The regional correlation coefficients, their associated standard error estimates, number of successful tests, and the order of magnitude of the minimum local $p$-value are shown in Table 1. Low-level, mid-level, high-level and total cloud correlations between SSN, NM2, AMO, and PDO are depicted in Figures 2-5, respectively.

Additionally, cross correlation coefficients were obtained for the dependent variables: solar proxies, GCR data, and climate indices; and for between altitudes regarding the regional cloud cover anomalies. The dependent variable time series cross correlation coefficients are presented in Table 2, and the cloud cover altitude regional cross correlation coefficients are presented in Table 3. Cross correlation coefficients between low-level cloud cover versus mid-level and high-level cloud cover are depicted in Figure 6.

The minimum local $p$-values were obtained to address the global field significance, also known as the multiplicity problem, which results from a large number of parallel statistical tests (Livezey \& Chen 1983). Additionally, climatological field variables exhibit high spatial autocorrelation, resulting in an increased probability of committing a Type 1 error, or falsely rejecting the global null hypothesis (Wilks 2006b). Walker's test (Wilks 2006a) was used to evaluate global field significance. Walker's minimum $p$-value is calculated:

$$
p_{\text {Walker }}=1-\left(1-\alpha_{\text {global }}\right)^{1 / K},
$$

where $K$ is the total number of parallel tests, and $\alpha_{\text {global }}$ represents the global confidence value $(0.05)$. Walker's test is robust against both the multiplicity test false discovery rate and the local spatial dependence (Wilks 2006a). The NARR domain used in this investigation is comprised of 277 by 349 gridpoints or 96,673 parallel statistical tests. Using a global significance level of $\alpha_{\text {global }}=0.05$, and $K=96,673$, provides a threshold $p_{\text {Walker }}$ of $5 \times 10^{-7}$. Tests, which have a $p_{\text {min }}$ value greater than or equal to the $p_{\text {walker }}$ threshold, fail to reject the global null hypothesis.

\section{Discussion}

\subsection{Regional correlations}

In general, the regionally averaged correlation coefficients only satisfy the $r_{\text {critical }}$ when separated into land or ocean subdomains. Additionally, there are many instances where the global null hypothesis cannot be rejected $\left(p_{\min } \geq p_{\text {Walker }}\right)$. For instance, the QBO fails to reject the global null hypothesis in all iterations of the correlation tests. Moreover, roughly half of all regionally averaged correlation tests do not satisfy the $r_{\text {critical }}$ of 0.096 . In Table 1, correlation tests, which do not meet 
Table 1. Pearson's Product-Moment correlation coefficients between NARR cloud cover detrended standard anomalies versus solar activity proxies, neutron monitors, and prominent climate indices.

\begin{tabular}{|c|c|c|c|c|c|c|c|c|c|c|c|c|}
\hline & \multicolumn{4}{|c|}{ Ocean } & \multicolumn{4}{|c|}{ Land } & \multicolumn{4}{|c|}{ All } \\
\hline & $r$ & $S E$ & $n$ & $p_{\text {min }}$ & $r$ & $S E$ & $n$ & $p_{\text {min }}$ & $r$ & $S E$ & $n$ & $p_{\text {min }}$ \\
\hline & \multicolumn{12}{|c|}{ Low cloud cover } \\
\hline SSN & -0.130 & 0.0008 & 28,511 & -16 & 0.125 & 0.0013 & 11.065 & -25 & -0.058 & 0.0009 & 39,576 & -25 \\
\hline FLUX & -0.130 & 0.0007 & 26,153 & -14 & 0.115 & 0.0014 & 10,138 & -24 & -0.062 & 0.0009 & 36,291 & -24 \\
\hline NM1 & 0.111 & 0.0008 & 21,640 & -10 & -0.122 & 0.0011 & 10,589 & -14 & 0.034 & 0.0009 & 32,229 & -14 \\
\hline NM2 & 0.119 & 0.0007 & 22,645 & -13 & -0.127 & 0.0011 & 10,028 & -13 & 0.043 & 0.0009 & 32,673 & -13 \\
\hline AMO & 0.093 & 0.0011 & 37,249 & -26 & -0.176 & 0.0007 & 21,268 & -24 & -0.005 & 0.0009 & 58,517 & -26 \\
\hline QBO & -0.027 & 0.0016 & 5388 & -5 & 0.098 & 0.0013 & 2062 & -3 & 0.007 & 0.0013 & 7450 & -5 \\
\hline ENSO & -0.118 & 0.001 & 28,251 & -24 & 0.096 & 0.0016 & 10,753 & -18 & -0.059 & 0.001 & 39,004 & -24 \\
\hline $\mathrm{NAO}$ & -0.056 & 0.0012 & 17,607 & -18 & 0.042 & 0.0013 & 17,683 & -26 & -0.007 & 0.0009 & 35,290 & -26 \\
\hline \multirow[t]{2}{*}{ PDO } & -0.102 & 0.001 & 36,564 & -19 & 0.138 & 0.0009 & 15,832 & -14 & -0.030 & 0.0009 & 52,396 & -19 \\
\hline & \multicolumn{12}{|c|}{ Mid cloud cover } \\
\hline SSN & 0.174 & 0.0003 & 33,036 & -13 & 0.172 & 0.0007 & 14,777 & -15 & 0.173 & 0.0003 & 47,813 & -15 \\
\hline FLUX & 0.175 & 0.0003 & 29,239 & -14 & 0.165 & 0.0008 & 13,207 & -15 & 0.172 & 0.0003 & 42,446 & -15 \\
\hline NM1 & -0.148 & 0.0002 & 28,421 & -6 & -0.168 & 0.0007 & 13,269 & -10 & -0.154 & 0.0003 & 41,690 & -10 \\
\hline NM2 & -0.154 & 0.0002 & 26,445 & -7 & -0.162 & 0.0006 & 13,289 & -11 & -0.157 & 0.0003 & 39,734 & -11 \\
\hline AMO & -0.223 & 0.0004 & 50,144 & -21 & -0.205 & 0.0005 & 23,380 & -19 & -0.218 & 0.0003 & 73,524 & -21 \\
\hline QBO & 0.117 & 0.0005 & 4395 & -3 & 0.105 & 0.0007 & 1451 & -2 & 0.114 & 0.0004 & 5846 & -3 \\
\hline ENSO & 0.141 & 0.0009 & 35,135 & -46 & 0.145 & 0.0009 & 16,629 & -13 & 0.142 & 0.0007 & 51,764 & -46 \\
\hline $\mathrm{NAO}$ & 0.093 & 0.001 & 17,459 & -23 & 0.086 & 0.0015 & 10,463 & -26 & 0.090 & 0.0008 & 27,922 & -26 \\
\hline \multirow[t]{2}{*}{ PDO } & 0.176 & 0.0005 & 43,454 & -18 & 0.155 & 0.0004 & 20,681 & -8 & 0.169 & 0.0004 & 64,135 & -18 \\
\hline & \multicolumn{12}{|c|}{ High cloud cover } \\
\hline SSN & 0.010 & 0.001 & 15,425 & -7 & 0.026 & 0.0012 & 15,739 & -11 & 0.018 & 0.0008 & 31,164 & -11 \\
\hline FLUX & 0.029 & 0.001 & 13,580 & -5 & 0.017 & 0.0013 & 14,118 & -11 & 0.023 & 0.0008 & 27,698 & -11 \\
\hline NM1 & -0.006 & 0.0012 & 10,727 & -7 & -0.037 & 0.0012 & 13,557 & -9 & -0.023 & 0.0009 & 24,284 & -9 \\
\hline NM2 & 0.001 & 0.0013 & 11,863 & -10 & -0.055 & 0.0013 & 13,135 & -9 & -0.028 & 0.0009 & 24,998 & -10 \\
\hline AMO & -0.109 & 0.0009 & 34,988 & -16 & -0.062 & 0.0011 & 21,416 & -15 & -0.091 & 0.0007 & 56,404 & -16 \\
\hline QBO & 0.092 & 0.0013 & 4103 & -4 & 0.094 & 0.0015 & 1841 & -3 & 0.093 & 0.001 & 5944 & -4 \\
\hline ENSO & -0.049 & 0.0012 & 35,597 & -27 & 0.120 & 0.0011 & 14,852 & -14 & 0.001 & 0.001 & 50,449 & -27 \\
\hline $\mathrm{NAO}$ & 0.036 & 0.0015 & 15,571 & -21 & -0.040 & 0.0017 & 9651 & -18 & 0.007 & 0.0012 & 25,222 & -21 \\
\hline \multirow[t]{2}{*}{ PDO } & -0.007 & 0.001 & 31,067 & -18 & 0.106 & 0.001 & 18,038 & -14 & 0.034 & 0.0008 & 49,105 & -18 \\
\hline & \multicolumn{12}{|c|}{ Total cloud cover } \\
\hline SSN & -0.125 & 0.0008 & 23,539 & -13 & 0.137 & 0.0012 & 11,909 & -22 & -0.037 & 0.0009 & 35,448 & -22 \\
\hline FLUX & -0.124 & 0.0008 & 21,436 & -12 & 0.130 & 0.0012 & 10,772 & -20 & -0.039 & 0.001 & 32,208 & -20 \\
\hline NM1 & 0.100 & 0.0008 & 18,400 & -10 & -0.140 & 0.0009 & 12,145 & -15 & 0.005 & 0.0009 & 30,545 & -15 \\
\hline NM2 & 0.113 & 0.0008 & 19,056 & -11 & -0.144 & 0.0008 & 12,282 & -12 & 0.013 & 0.0009 & 31,338 & -12 \\
\hline AMO & 0.012 & 0.0011 & 34,520 & -22 & -0.194 & 0.0005 & 22,318 & -19 & -0.069 & 0.0008 & 56,838 & -22 \\
\hline QBO & 0.008 & 0.0016 & 4797 & -3 & 0.098 & 0.0011 & 2003 & -3 & 0.034 & 0.0013 & 6800 & -3 \\
\hline ENSO & -0.095 & 0.0012 & 27,845 & -24 & 0.135 & 0.0013 & 12,911 & -20 & -0.022 & 0.001 & 40,756 & -24 \\
\hline NAO & -0.029 & 0.0014 & 15,977 & -19 & 0.076 & 0.0012 & 16,245 & -24 & 0.024 & 0.001 & 32,222 & -24 \\
\hline PDO & -0.058 & 0.001 & 30,319 & -17 & 0.159 & 0.0006 & 18,348 & -14 & 0.024 & 0.0008 & 48,667 & -17 \\
\hline
\end{tabular}

SSN and FLUX refer to sun spot number and solar radio flux, respectively. North American GCR neutron data for Newark, New Jersey (NM1) and Thule Air Force base, Greenland (NM2). Climatological indices included in the correlation matrix are the Atlantic Multidecadal Oscillation (AMO), the Quasi-Biennial Oscillation (QBO), the multivariate El Nino Southern Oscillation (ENSO), the North Atlantic Oscillation (NAO), and the Pacific Decadal Oscillation (PDO). Correlation coefficients $(r)$ represent ocean, land, and total domain averages, the standard error $(S D), n$ : number of significant $p$-values, and the order of magnitude of the minimum $p$-value for field significance testing. Walker's test for global field significance requires a minimum $p$-value to be no greater than $10^{-7}$.

the $r_{\text {critical }}$ or fail to pass Walker's minimum $p$-value test, are shaded in gray.

\subsubsection{Low cloud cover correlations}

For low cloud cover, only the solar proxies, GCR proxies, the AMO, PDO, and ENSO exhibit significant correlation coefficients, and no correlation variable satisfies the $r_{\text {critical }}$ for cloud cover when averaged over the entire domain. There exists a unique asymmetry for with low cloud cover, specifically regarding a significant negative (positive) correlation between low level ocean cloud with solar proxies (GCR proxies) and a positive (negative) correlation with land cloud cover. The correlation coefficients between solar proxies and low level maritime cloud cover are the highest, with coefficients of -0.130; AMO correlates with low level continental cloud cover at -0.176 , which is the highest coefficient for low level continental cloud cover.

Regionally, for SNN and NM2 (Figs. 2 and 3, respectively) there exists a very sharp distinction between correlation over the ocean versus land. For the AMO, the correlation coefficients are almost entirely negative over land, whereas the ocean correlation does exhibit negative coefficients' cluster in the Central Pacific flanked by positive correlation coefficients. 
D.S. Krahenbuhl: Investigating a solar influence on cloud cover using the NARR data
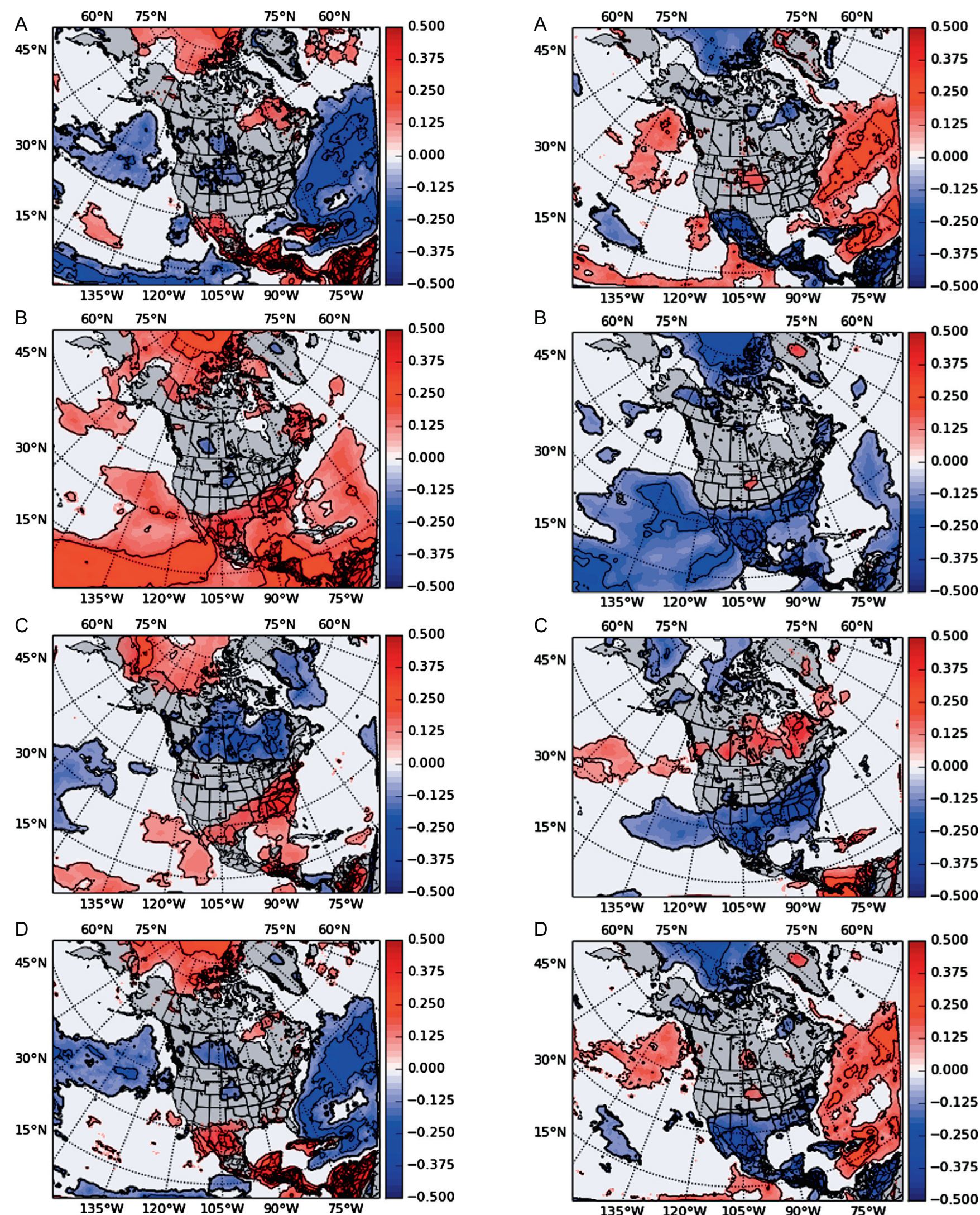

Fig. 2. Regional Pearson's $R$ correlation between Sun Spot Number (SSN) and cloud cover deseasonalized standard anomalies for A: low, B: mid, C: High, D: total cloud cover. Regions not satisfying a $p$-value less than .05 were standardized to $r=0$. Red (blue) shading indicates areas of positive (negative).

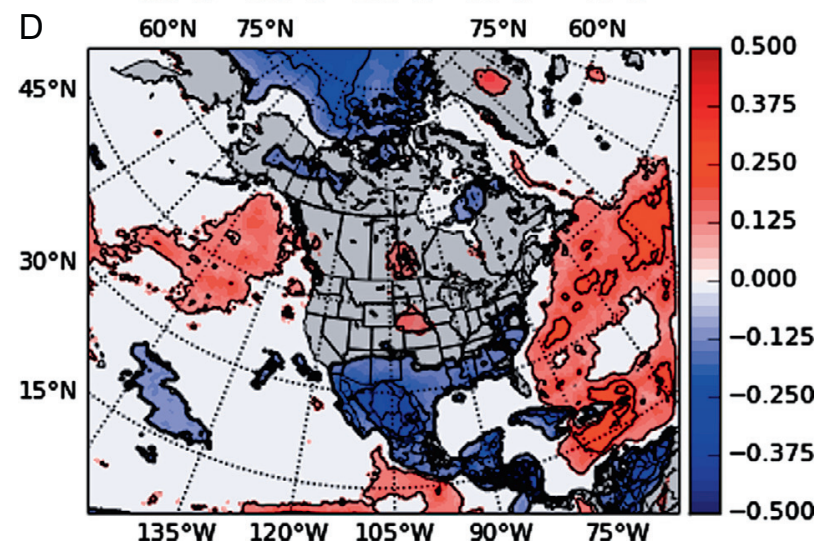

Fig. 3. Regional Pearson's $R$ correlation between Thule Air Force Base Neutron Monitor (NM2) and cloud cover deseasonalized standard anomalies for A: low, B: mid, C: High, D: total cloud cover. Regions not satisfying a $p$-value less than .05 were standardized to $r=0$. Red (blue) shading indicates areas of positive (negative) correlation. 
J. Space Weather Space Clim., 5, A11 (2015)

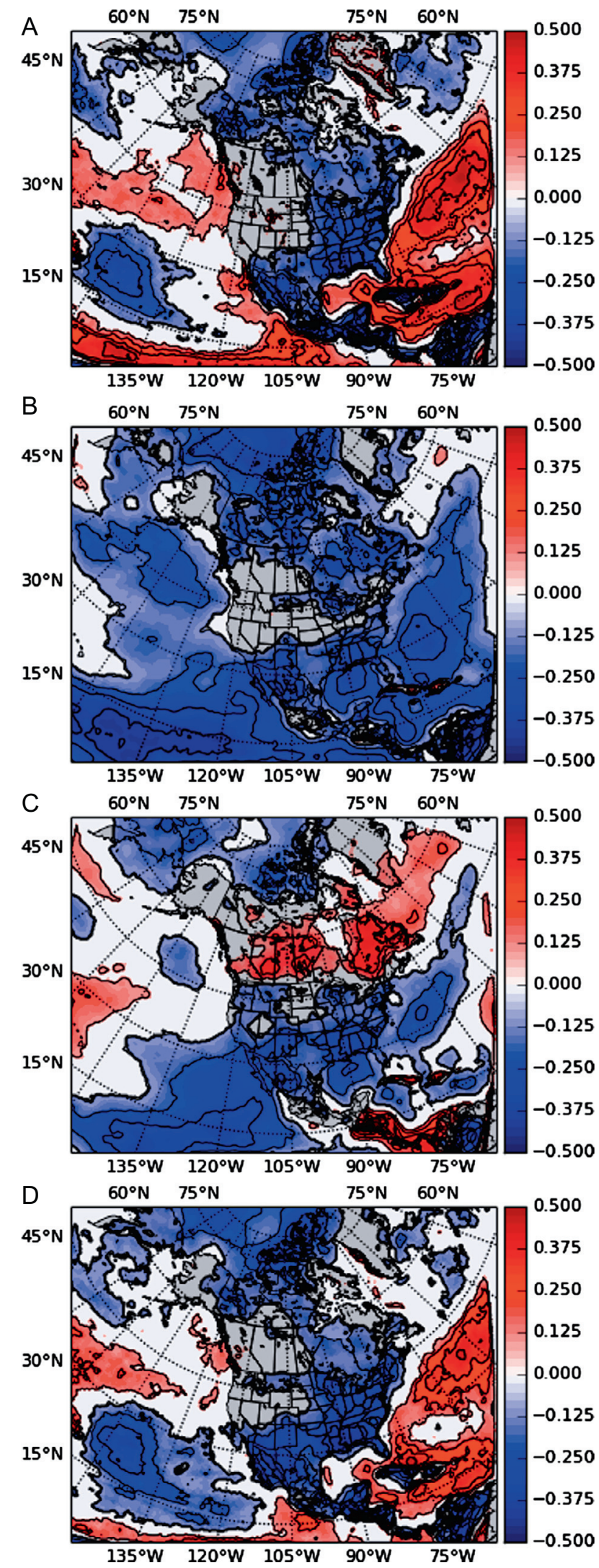

Fig. 4. Regional Pearson's $R$ correlation between the Atlantic Multidecadal Oscillation (AMO) and cloud cover deseasonalized standard anomalies for A: low, B: mid, C: High, D: total cloud cover. Regions not satisfying a $p$-value less than .05 were standardized to $r=0$. Red (blue) shading indicates areas of positive (negative) correlation.
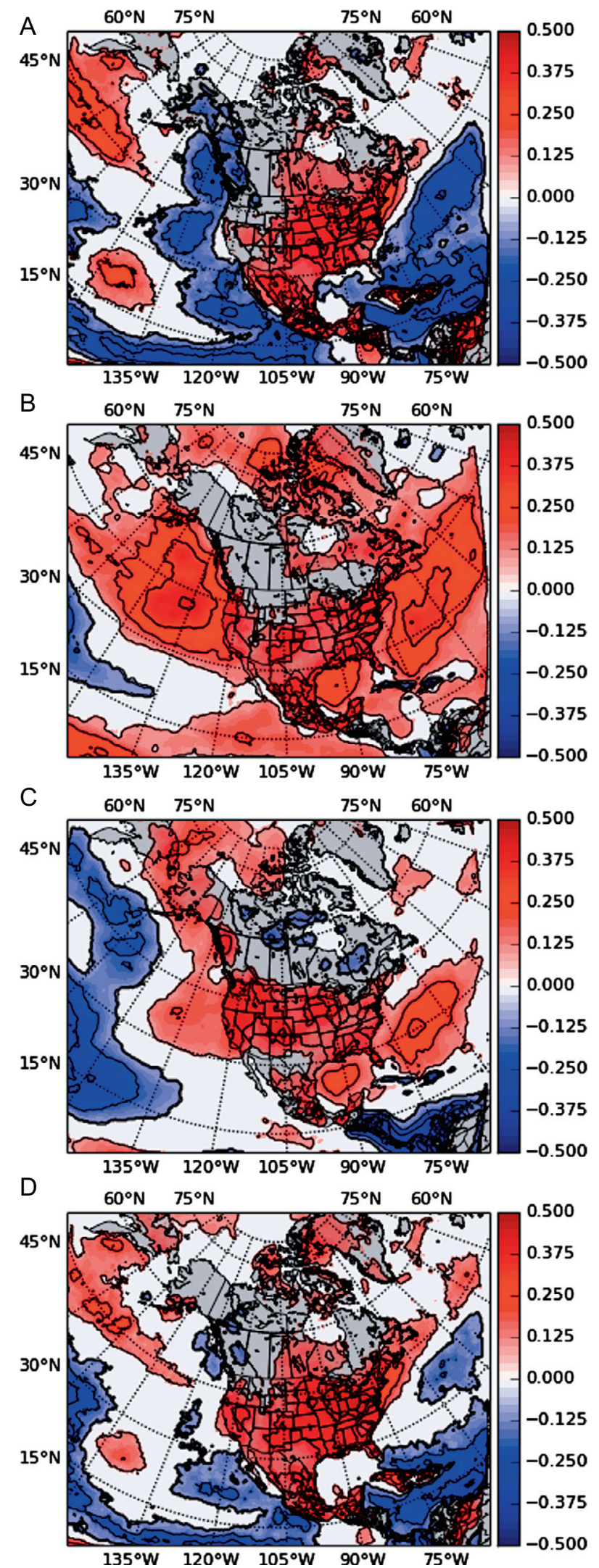

Fig. 5. Regional Pearson's $R$ correlation between the Pacific Decadal Oscillation (PDO) and cloud cover deseasonalized standard anomalies for A: low, B: mid, C: High, D: total cloud cover. Regions not satisfying a $p$-value less than .05 were standardized to $r=0$. Red (blue) shading indicates areas of positive (negative) correlation. 
D.S. Krahenbuhl: Investigating a solar influence on cloud cover using the NARR data

Table 2. Pearson Product-Moment Cross Correlation between dependent variables.

\begin{tabular}{|c|c|c|c|c|c|c|c|c|c|}
\hline & SSN & $\mathrm{FX}$ & NM1 & NM2 & $\mathrm{AMO}$ & QBO & ENSO & NAO & PDO \\
\hline SSN & 1.000 & 0.972 & -0.796 & -0.767 & -0.190 & -0.006 & 0.021 & 0.153 & -0.094 \\
\hline FX & 0.972 & 1.000 & -0.800 & -0.791 & -0.169 & 0.011 & -0.005 & 0.148 & -0.144 \\
\hline NM1 & -0.796 & -0.800 & 1.000 & 0.973 & 0.239 & 0.030 & -0.082 & -0.182 & 0.016 \\
\hline NM2 & -0.767 & -0.791 & 0.973 & 1.000 & 0.206 & 0.021 & -0.079 & -0.179 & 0.011 \\
\hline AMO & -0.190 & -0.169 & 0.239 & 0.206 & 1.000 & -0.076 & -0.089 & -0.246 & -0.221 \\
\hline QBO & -0.006 & 0.011 & 0.030 & 0.021 & -0.076 & 1.000 & 0.023 & 0.017 & 0.011 \\
\hline ENSO & 0.021 & -0.005 & -0.082 & -0.079 & -0.089 & 0.023 & 1.000 & -0.002 & 0.516 \\
\hline NAO & 0.153 & 0.148 & -0.182 & -0.179 & -0.247 & 0.017 & -0.002 & 1.000 & 0.019 \\
\hline PDO & -0.094 & -0.144 & 0.016 & 0.011 & -0.221 & 0.011 & 0.516 & 0.019 & 1.000 \\
\hline
\end{tabular}

SSN and FLUX refer to sun spot number and solar radio flux, respectively. North American GCR neutron data for Newark, New Jersey (NM1) and Thule Air Force base, Greenland (NM2). Climatological indices included in the correlation matrix are the Atlantic Multidecadal Oscillation (AMO), the Quasi-Biennial Oscillation (QBO), the multivariate El Nino Southern Oscillation (ENSO), the North Atlantic Oscillation (NAO), and the Pacific Decadal Oscillation (PDO). Bold coefficients indicate $p$-vales less than 0.05 .

Table 3. Regional Cross Correlation between NARR cloud cover detrended standard anomalies.

\begin{tabular}{|c|c|c|c|c|c|c|c|c|c|c|c|c|}
\hline & \multicolumn{4}{|c|}{ Ocean } & \multicolumn{4}{|c|}{ Land } & \multicolumn{4}{|c|}{ All } \\
\hline & $r$ & $S E$ & $n$ & $p_{\min }$ & $r$ & $S E$ & $n$ & $p_{\min }$ & $r$ & $S E$ & $n$ & $p_{\min }$ \\
\hline LCDC vs. MCDC & 0.071 & 0.0014 & 40,490 & -118 & 0.510 & 0.0010 & 32,597 & -174 & 0.267 & 0.0012 & 73,087 & -174 \\
\hline LCDC vs. HCDC & -0.035 & 0.0011 & 34,080 & -33 & 0.232 & 0.0014 & 23,130 & -92 & 0.073 & 0.0010 & 57,210 & -92 \\
\hline LCDC vs. TCDC & 0.856 & 0.0002 & 61,958 & -313 & 0.876 & 0.0008 & 32,731 & $\sim-100$ & 0.863 & 0.0003 & 94,686 & $\sim-100$ \\
\hline MCDC vs. HCDC & 0.624 & 0.0005 & 61,944 & -134 & 0.596 & 0.0007 & 32,987 & -203 & 0.614 & 0.0004 & 94,931 & -203 \\
\hline MCDC vs. TCDC & 0.405 & 0.0006 & 59,274 & -185 & 0.706 & 0.0009 & 33,023 & $\sim-100$ & 0.513 & 0.0007 & 92,297 & $\sim-100$ \\
\hline HCDC vs. TCDC & 0.375 & 0.0006 & 61,510 & -182 & 0.473 & 0.0013 & 30,908 & -315 & 0.407 & 0.0006 & 92,418 & -315 \\
\hline
\end{tabular}

LCDC: low cloud cover, MCDC: mid-level cloud cover, HCDC: high-level cloud cover. Correlation coefficients $(r)$ represent ocean, land, and total domain averages, the standard error $(S D)$, number of significant $p$-values, and the order of magnitude of the minimum $p$-value for field significance testing. Walker's test for global field significance requires a minimum $p$-value to be no greater than $10^{-7}$.

Interestingly, low cloud cover pattern for the PDO is almost inverse of the AMO.

\subsubsection{Mid-level cloud cover correlations}

Mid-level cloud cover is the only altitude which maintains correlation values above the critical value when averaged over the entire domain (see Table 1). Interestingly, both GCR proxy correlations failed to reject the global null hypothesis for midlevel maritime cloud cover, whereas solar radio flux shows a positive correlation coefficient of 0.175 . However, the asymmetry between land and ocean is absent here for solar proxies, as SSN and FX exhibit $\sim 0.17$ for continental, maritime, and total mid-level cloud cover. The inverse relationship folds for GCRs vs. solar having negative forcings over land and the entire domain. Additionally, the ENSO and PDO show consistent positive correlation coefficients $(\sim 0.142$ and $\sim 0.165$, respectively) at all subdomains at this mid-level cloud altitude. However, the AMO exhibits the highest magnitude correlations for all subdomains of mid-level cloud cover having negative correlation coefficients of $-0.223,-0.205$, and -0.218 for ocean, land, and entire domain respectively.

Regionally, in Figure 2, SSN exhibits widespread positive correction in the tropics to subtropics and the Polar Regions. This is essentially inverse in Figure 3, where NM2 exhibits the opposite pattern. Whereas, the negative correlation coefficients between the AMO and mid-level cloud cover (Fig. 4) all but covers the entire map. Again, it appears that the PDO correlation exhibits the opposite pattern of the AMO, portraying positive correlation coefficients across the entire domain.

\subsubsection{High cloud cover correlations}

High-level cloud cover exhibited the least amount of valid correlation tests of any cloud cover altitude. Solar or GCR proxy correlation did not yield an absolute correlation coefficient greater than $r_{\text {critical }}$. The AMO exhibits the strongest correlation coefficients over the oceans at -0.109 , however it does not yield a valid coefficient for land or the entire domain. The only variables, which provide a valid correlation coefficient over continental high clouds, are the ENSO at 0.120 and the PDO at 0.106. As with low level and total level cloud cover, no variable achieves a valid correlation coefficient over the entire domain for high cloud cover.

However, there appears to be a noticeable latitudinal asymmetry for high cloud cover correlation coefficients across both ocean and land subdomains, possibly resulting in these subcritical correlation coefficients. For SSN, there are marked positive correlation coefficients over the tropics and the United States Gulf Coast and Eastern Seaboard with negative correlation coefficients over much of Canada, Greenland, and the North Central Pacific. For NM1, there exists an almost reciprocal pattern: negative correlation coefficients near the South US and Atlantic Seaboard, with positive correlation coefficients in Canada. This NM1 correlation is mirrored in the AMO high cloud correlation pattern. Given that the NARR high-level 


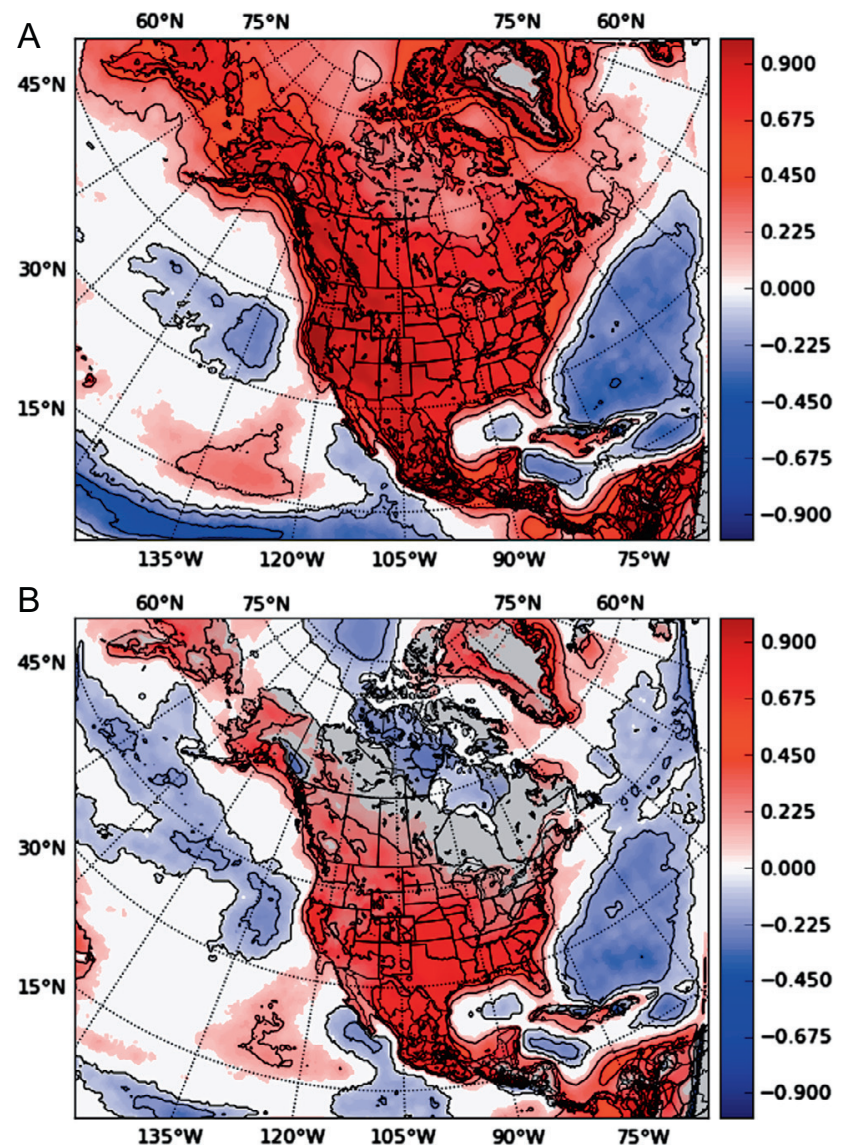

Fig. 6. Cross correlation between (a) Low cloud cover anomalies versus mid-level cloud cover anomalies and (b) Low cloud cover anomalies versus high-level cloud cover anomalies. Regions not satisfying a $p$-value less than .05 were standardized to $r=0$. Red (blue) shading indicates areas of positive (negative) correlation.

cloud layer is situated above $350 \mathrm{mb}$, this latitudinal asymmetry is likely due to the positioning of the subtropical and polar jet streams.

\subsubsection{Total cloud cover correlations}

The total cloud cover layer exhibits very similar patterns to low-level cloud cover. For maritime total cloud cover, SSN has a strong -0.125 correlation coefficient. For continental cloud cover, the AMO has a -0.194 correlation coefficient. However, the ENSO total cloud cover correlations more accurately reflect its mid-level and high-level cloud cover patterns for land, with a correlation coefficient of 0.135 . Continental total cloud cover has similarities to the PDO low-level, midlevel, and high clouds at 0.159 . Regional correlation patterns of total cloud cover closely mirror low-level cloud cover.

\subsection{Cross correlation}

Cross correlation coefficients between dependent variable time series are displayed in Table 2. Significant correlation coefficients $(p \leq 0.05)$ are in bold type. The AMO exhibits positive correlation coefficients with GCR NM1 and NM2 at 0.239 and 0.206 , respectively; and negative correlation coefficients between with SSN and FX at -0.190 , and -0.170 , respectively. The AMO additionally exhibits a negative correlation coefficient with the NAO and the PDO at -0.247 , and
-0.221 , respectively. The QBO presents significant correlation with the solar proxies, GCR proxies, or any other climate index. The ENSO's only significant relation is a large positive correlation coefficient with the PDO at 0.516. The NAO displays positive correlation coefficients with SSN and FX at 0.135 , and 0.148 respectively; and negative correlation coefficients between NM1 and NM2 at -0.182 and -0.179 , respectively. Interestingly, the PDO displays a negative correlation coefficient with FX at -0.144 , but not significant with SSN, NM1 or NM2.

For the most part, there is widespread positive cross correlation between cloud cover at different altitudes over land, ocean, and total (Table 3 ). This conflicts with previous findings which utilize satellite observations which find widespread negative correlation between low and middle to high cloud cover. However, a major exception occurs for ocean low cloud cover cross correlation with medium and high ocean cloud cover. Low ocean cloud cover fails to produce a correlation coefficient larger than the $r_{\text {critical }}$ value between other ocean cloud altitudes. It is the author's assumption that the assimilation reanalysis process is responsible for the widespread positive correlations between cloud cover altitudes, however further investigation is required. The cross correlation coefficients additionally indicate that low cloud cover, over land, ocean, and the entire domain exert the most control over the total cloud cover: $0.856,0.876$, and 0.863 , respectively.

\subsection{Previous findings}

For GCRs, this investigation does find a positive correlation between GCRs and low-level marine cloud cover particularly in the mid-latitude oceans, consistent with previous findings (Svensmark \& Friis-Christensen 1997; Marsh \& Svensmark 2000; Yu 2002; Voiculescu et al. 2006; Usoskin \& Kovaltsov 2008). It should be noted, however, that SSN and FX exhibit slightly larger correlation coefficients than NM1 and NM2, -0.130 and -0.130 versus 0.111 and 0.119 , respectively. This investigation also finds a negative correlation between GCRs and land low cloud cover in agreement with Balling \& Cerveny (2003) albeit for different reasons (the majority of the land correlations are located in Central America, not in the US). However, the AMO correlation of -0.176 is the greatest correlation magnitude for low land cloud cover.

Regional correlation for high cloud cover cannot be compared to previous work due to their latitudinal asymmetry, which resulted in subcritical correlation coefficients. Additionally, maritime high cloud cover failed to reject the global null hypothesis for field significance. The spatial distribution of GCR correlation with high cloud (Fig. 3) shows a plume of negative correlation covering much of the southern to northeastern United States. However, between $35^{\circ}$ and $60^{\circ}$ north, a positive correlation exists over parts of the Pacific and much of Canada.

\section{Conclusions}

For low cloud cover, the regional correlations are consistent with previous research. Low cloud cover over the North American continental interior and for regions of the midlatitude oceans exhibited a positive correlation with cosmic ray flux (Svensmark \& Friis-Christensen 1997; Marsh \& Svensmark 2000; Yu 2002; Voiculescu et al. 2006; Usoskin \& Kovaltsov 2008). Additionally, this maritime low cloud 
cover exhibits the only failed correlation significance with other altitudes. The cross correlation reveals that cloud cover is positively correlated everywhere but for ocean low cloud cover, supporting the unique response of the marine layer. However, the solar proxies registered a slightly higher regional correlation coefficient than the GCRs. Additionally, low cloud cover exhibited noticeable asymmetry between land/ocean and Solar/ GCRs. Further, extensive positive cross correlation between cloud cover altitudes is anomalous with regard to previous investigations. It is unclear whether the reanalysis process is responsible.

Out of all the correlation analyses, the QBO proxies exhibit the least remarkable correlation with cloud cover, failing to reject the global null hypothesis in all test iterations. The Atlantic Multidecadal Oscillation exhibited the maximum influence on cloud cover, was negatively correlated with cloud cover at all levels over all surfaces which satisfied $r_{\text {critical }}$. The ENSO and PDO exhibited the subsequent highest correlations, with regional correlation coefficients similar to SSN/FX, and NM1/NM2. The ENSO and PDO additionally exhibited the greatest cross correlation coefficients at 0.516 .

In regard to the solar forcing of the climate system, the results of this investigation suggest that with the assumption that solar forcing does impact cloud cover, measurements of solar activity exhibit a slightly higher correlation than GCRs. The only instance where GCRs exhibit a positive regional correlation with cloud cover is for maritime low clouds.

Several researchers (Farrar 2000; Roy \& Haigh 2010) argue that Pacific Ocean cloud cover correlations with GCRs are coincident with the ENSO. This investigation's results find that the regional correlation of the ENSO is negative for low maritime cloud cover (opposite of GCRs). Additionally, the ENSO time series failed to produce a significant cross correlation coefficient between solar proxies or GCR proxies (Table 2). However, the cloud cover correlations could possibly be coincident with other climate indices (Laken et al. 2012b) with the AMO, which not only exhibits the largest influence on regional cloud cover, but also displays significant correlations with both solar proxies and GCR proxies. Other researchers have raise the possibility that long-term solar activity may act as a chaotic attractor for the various climatological modes of the atmosphere and ocean, and that they may even exhibit chaotic synchronization at times (Palus et al. 2007). Although both solar activity and GCR proxies exhibit regional correlations, specifically over marine low clouds, the AMO appears to exert the greatest control of cloud cover in the NARR domain.

Acknowledgements. The author thanks the curators of the NMDB database (www.nmdb.eu) founded under the European Union's FP7 programme (Contract No. 213007), and the PIs of individual neutron monitor data. The author thanks the Earth Science Research Laboratory, Physical Science Division with the National Oceanic and Atmospheric Administration, located at Boulder, Colorado for the solar activity, and climate data. Additionally, the author wishes to thank the dedicated, supportive peer reviewers for their insightful, constructive suggestions, and guidance. The editor thanks $\mathrm{M}$. Voiculescu and one anonymous referee for their assistance in evaluating this paper.

\section{References}

Arnold, F. Atmospheric Aerosol and cloud condensation nuclei formation: a possible influence of cosmic rays? Space Sci. Rev., 125 (1-4), 169-186, 2006.
Balling Jr., R.C., and R.S. Cerveny. Cosmic ray flux impact on clouds? An analysis of radiosondes, cloud cover, and surface temperature records from the United States. Theor. Appl. Climatol., 75, 225-231, 2003.

Čalogović, J., C. Albert, F. Arnold, J. Beer, L. Desorgher, and E.O. Flueckiger. Sudden cosmic ray decreases: no change of global cloud cover. Geophys. Res. Lett., 37, L03802, 2010, DOI: 10.1029/2009GL041327.

Dunne, E., L. Lee, C. Reddington, and K.S. Carslaw. No statistically significant effect of a short-term decrease in the nucleation rate on atmospheric aerosols. Atmos. Chem. Phys., 12 (23), 11573-11587, 2012.

Erlykin, A.D., G. Gyalai, K. Kudela, T. Sloan, and A.W. Wolfendale. Some aspects of ionization and the cloud cover, cosmic ray correlation problem. J. Atmos. Sol. Terr. Phys., 71 (8-9), 823-829, 2009a, DOI: 10.1016/j.jastp.2009.03.007.

Erlykin, A.D., T. Sloan, and A.W. Wolfendale. The search for cosmic ray effects on clouds. J. Atmos. Sol. Terr. Phys., 71 (8-9), 955-958, 2009b, DOI: 10.1016/j.jastp.2009.03.019.

Erlykin, A.D., G. Gyalai, K. Kudela, T. Sloan, and A.W. Wolfendale. On the correlation between cosmic ray intensity and cloud cover. J. Atmos. Sol. Terr. Phys., 71, 1794-1806, 2009c, DOI: $10.1016 /$ j.jastp.2009.06.012.

Erlykin, A., T. Sloan, and A. Wolfendale. Correlations of clouds, cosmic rays and solar irradiation over the Earth. J. Atmos. Sol. Terr. Phys., 72, 151-156, 2010, DOI: $10.1016 /$ j.jastp.2009.11.002.

Erlykin, A., T. Sloan, and A. Wolfendale. A review of the relevance of the "CLOUD" results and other recent observations to the possible effect of cosmic rays on the terrestrial climate. Meteorol. Atmos. Phys., 121, 137-142, 2013,

DOI: $10.1007 / \mathrm{s} 00703-013-0260-\mathrm{x}$.

Farrar, P. Are cosmic rays influencing oceanic cloud coverage - or is it only el nino? Clim. Change, 47, 7-15, 2000.

Kazil, J., K. Zhang, P. Stier, J. Feichter, U. Lohmann, and K. O'Brien. The present-day decadal solar cycle modulation of Earth's radiative forcing via charged $\mathrm{H} 2 \mathrm{SO} 4 / \mathrm{H} 20$ aerosol nucleation. Geophys. Res. Lett., 39, 2, 2012.

Kirkby, J., J. Curtius, J. Almeida, E. Dunne, and J. Duplissy, et al. Role of sulphuric acid, ammonia and galactic cosmic rays in atmospheric aerosol nucleation. Nature, 476, 429-433, 2011.

Kristjansson, J.E., A. Staple, and J. Kristiansen. A new look at possible connections between solar activity, clouds and climate. Geophys. Res. Lett., 29, 2017, 2002.

Kristjansson, J., J. Kristiansen, and E. Kaas. Solar activity, cosmic rays, clouds and climate - an update. Adv. Space Res., 34, 407-415, 2004

Kristjannson, J., C.W. Stjern, F. Stordal, A.M. Fjaeraa, G. Myhre, and $\mathrm{K}$. Jonasson. Cosmic rays, cloud condensation nuclei and clouds - a reassessments using MODIS data. Atmos. Chem. Phys., 8, 7373-7387, 2008.

Laken, B.A., and J. Čalogović. Solar irradiance, cosmic rays and cloudiness over daily timescales. Geophys. Res. Lett., 38, L24811, 2011, DOI: 10.1029/2011GL048764.

Laken, B.A., and J. Calogović. Composite analysis with Monte Carlo methods: an example with cosmic rays and clouds. J. Space Weather Space Clim., 3, A29, 2013.

Laken, B.A., and D. Kniveton. Forbush decreases and Antarctic cloud anomalies in the upper troposphere. J. Atmos. Sol. Terr. Phys., 73, 371-376, 2011, DOI: 10.1016/j.jastp.2010.03.008.

Laken, B., D. Kniveton, and A. Wolfendale. Forbush decreases, solar irradiance variations, and anomalous cloud changes. J. Geophys. Res. [Atmos.], 116, D09201, 2011, DOI: 10.1029/2010JD014900.

Laken, B.A., E. Palle, J. Čalogović, and E.M. Dunne. A cosmic rayclimate link and cloud observations. J. Space Weather Space Clim., 2, A18, 2012a, DOI: 10.1051/swsc/2012018.

Laken, B.A., E. Palle, and H. Miyahara. A decade of the moderate resolution imaging spectroradiometer: is a solar cloud link detectable? J. Clim., 25 (13), 4430-4440, 2012b. 
Laut, P. Solar activity and terrestrial climate: an analysis of some purported correlations. J. Atmos. Sol. Terr. Phys., 65, 801-812, 2003

Lean, J., and D. Rind. Climate forcing by changing solar radiation. J. Clim., 11, 3069-3094, 1998.

Livezey, R.E., and W.Y. Chen. Statisical field significance and its determination by Monte Carlo techniques. Monthly Weather Review, 111, 46-59, 1983.

Marsh, N., and H. Svensmark. Cosmic rays, clouds and climate. Space Sci. Rev., 94, 215-230, 2000.

Mesinger, F., G. DiMego, E. Kalnay, K. Mitchel, P.C. Shafran, et al. North American Regional Reanalysis. Bull. Am. Meteorol. Soc., 87 (3), 343-360, 2006

Palle, E. Possible satellite perspective effects on the reported correlations between solar activity and clouds. Geophys. Res. Lett, 32, L03802, 2005.

Palus, M., J. Kurths, U. Schwarz, N. Seehafer, D. Novotna, and T. Charvatova. The Solar Activity Cycle is Weakly Synchronized with the Solar Inertial Motion. Phys. Lett. A, 365 (5-6), 421-428, 2007, DOI: 10.1016/j.physleta.2007.01.039.

Roy, I., and J. Haigh. Solar cycle signals in sea level pressure and sea surface temperature. Atmos. Chem. Phys., 10, 3147-3153, 2010.

Snow-Kropla, E., J. Pierce, D. Westervelt, and W. Trivitayanurak. Cosmic rays, aerosol formation and cloud-condensation nuclei: sensitivities to model uncertainties. Atmos. Chem. Phys., 11 (8), 4001-4013, 2011
Svensmark, H. Cosmic rays and earth's climate Space Sci. Rev., 155-166, 2000.

Svensmark, H., and E. Friis-Christensen. Variation of cosmic ray flux and global cloud coverage - a missing link in solar-climate relationships. J. Atmos. Sol. Terr. Phys., 59, 1225-1232, 1997.

Udelhofen, P., and R. Cess. Cloud cover variations over the United States: An influence of cosmic rays or solar variability? Geophys. Res. Lett., 28, 2517-2620, 2001.

Usoskin, I.G., and G. Kovaltsov. Cosmic rays and climate of the Earth: possible connection. C.R. Geosci., 340, 441-450, 2008.

Voiculescu, M., and I. Usoskin. Persistent solar signatures in cloud cover: spatial and temporal analysis. Environ. Res. Lett., 7, 044004, 2012.

Voiculescu, M., I. Usoskin, and K. Mursula. Different response of clouds to solar input, Geophys. Res. Lett., 33, L21802, 2006.

Wilks, D.S. On "Field Significance", and the false discovery rate. Journal of Applied Meteorology and Climatology, 45, 1181-1189, 2006a.

Wilks, D.S. Statistical methods in the atmosphere sciences, $\mathbf{1 0 0}$, 170-176, 2006b.

Yu, F. Altitude variations of cosmic ray induces production of aerosols: implications for global cloudiness and climate. J. Geophys. Res., 107, 1118, 2002.

Zhao, Q., T. Black, and M. Baldwin. Implementation of the cloud prediction scheme in the Eta model at NCEP, Weather Forecast, $697-712,1997$.

Cite this article as: Krahenbuhl D.S. Investigating a solar influence on cloud cover using the North American Regional Reanalysis data. J. Space Weather Space Clim., 5, A11, 2015, DOI: 10.1051/swsc/2015012. 